\title{
Antiphospholipid syndrome in Mexican children: evolution, laboratory and clinical characteristics: a I0-year experience
}

\section{Introduction}

The Antiphospholipid syndrome (APS) is a multisystem and autoimmune disease, which is mainly characterized by the presence of thrombotic events, gestational morbidity, as well as hematological, dermatological and neurological manifestations, in the presence of high titers of Antiphospholipid antibodies. ${ }^{1}$ It can present as a primary entity or secondary to another autoimmune disease, mainly systemic lupus erythematosus. After 35 years of its first description, the understanding of this pathology is still evolving and even more in its presentation in the pediatric patients. The presence of Antiphospholipid antibodies has been widely reported in pediatric patients with thrombosis, and less frequently in isolated hematologic and neurological manifestations. APS is considered the most common acquired cause of a prothrombotic state of autoimmune etiology. At the moment, there are no reliable data on the incidence or prevalence of this syndrome in pediatrics. Although the incidence of thrombosis in children is lower than in adults, the thrombosis attributable to Antiphospholipid antibodies is proportionately greater. The longterm morbidity and mortality associated with thrombosis events in pediatric population may be minored by predict which children are at higher risk of thrombosis associated to APS, sought to determine prognosis and select patients for prophylactic treatment and more rigorous follow-up.

\section{Objectives}

To describe the clinical presentation and evolution in addition to laboratory findings in Mexican pediatric population who developed arterial o venous thrombosis in the presence of high titers of Antiphospholipid antibodies. Retrospective cohort study of the Children's Hospital of Mexico Federico Gomez, last 10 years.

\section{Method}

Retrospective cohort study of the Children's Hospital of Mexico Federico Gomez, last 10 years. We reviewed the data from the clinical archives of the patients with diagnosis of APS according to the Miyakis criteria, from 2007 to 2017. The variables analyzed include age at diagnosis, sex, subtype of APS, clinical finding of thrombosis, laboratory finding of Antiphospholipid antibodies and outcome.

\section{Results}

A total of 29 patients fulfill the diagnosis criteria of APS. The mean age of patients at diagnostic was 9.8 years, with a minor age at diagnosis of 2.2 years and a maximum of 16.4 years of age. Of the total population $52 \%$ were females and $48 \%$ males. Primary APS was diagnosed in $48 \%$, of which $71 \%$ were males. Secondary APS was present in $52 \%$ of the population; all diagnosed with Systemic Lupus Erythematosus. Of the patients with secondary APS, 85\% were female. Arterial thrombosis was present in $48 \%$ of the cases, primarily in the group of secondary APS..$^{2-5}$ Of the total cases $20 \%$
Volume 9 Issue 2 - 2019

\author{
M Brana-Ruiz, L Aparicio, S Osorio,A \\ Benitez,V Cortez, M Gomez,A Rodriguez, E \\ Faugier, R Maldonado \\ Pediatric Rheumatology, Hospital Infantile de Mexico Federico \\ Gomez, Mexico
}

\author{
Correspondence: Maria Teresa BrañaRuiz, Designation \\ Pediatric Rheumatology, University Hospital Infantil de Mexico \\ Federico Gomez, Dr. Marquez 162, Colonia Doctores. Del. \\ Cuahutemoc. 06720, Mexico, Tel 52 I55 595I5696, \\ Emailmaritebranaruiz@gmail.com
}

Received: September II, 2018 | Published: March 19, 2019

presents with a second thrombosis event despite anticoagulant therapy, of this $66 \%$ were diagnosed with secondary APS. Lupus anticoagulant (LA) was present in high titers in $93 \%$ of the patients, while anti-B2 glycoprotein-1 antibody IgG was present in $27 \%$ and IgM in $31 \%$. Anticardiolipin antibody IgG was positive in $17 \%$ of the population, while isotope IgM in $24 \%$. All patients receive long term anticoagulant therapy. One male patient, with secondary APS developed catastrophic APS. One patient required of amputation for deep venous thrombosis, and another patient died as a complication of gastrointestinal thrombosis, both males with primary APS.

\section{Conclusion}

During a 10 year follow up, we diagnosed 29 patients with APS. As reported in literature, a greater percentage of patients are female and present with a secondary APS. Most patients with primary APS are males. In our population, in contrast with what literature report, secondary APS presents with a greater percentage of arterial thrombosis. The mayor site of venous thrombosis was low extremities and of arterial thrombosis were cerebrovascular events. The presence of positive LA implicates a higher risk of thrombosis. Our pediatric population diagnosed with APS requires of a close follow up in order to monitor anticoagulant therapy and to prevent the patients from developing a second thrombotic event that may lead to death.

\section{Acknowledgments}

None.

\section{Conflicts of interest}

The authors declared there is no conflicts of interest.

\section{References}

1. Petty RE, Lindsley CB. Cassidys textbook of pediatric rheumatology. 7th ed. Philadelphia, PA: Elsevier; 2016.

2. Miyakis $\mathrm{S}$, Lockshin $\mathrm{MD}$, Atsumi $\mathrm{T}$, et al. International consensus statement on an update of the classification criteria for definite 
antiphospholipid syndrome (APS). J Thromb Haemost. 2006;4(2):295306.

3. R Cervera, J Piette, J Font et al. Antiphospholipid syndrome clinical and immunologic manifestations and patterns of disease expression in a cohort of 1,000 patients, Arthritis Rheum. 2002;46(4):1019-1027.
4. B Tavil, E Ozyurek, F Gumruk, et al. Antiphospholipid antibodies in Turkish children with thrombosis, Blood Coagul Fibrinolysis. 2007;18(4):347-352.

5. T Avcin, R Cimaz, ED Silverman, et al. Pediatric Antiphospholipid syndrome: clinical and immunologic features of 121 patients in an international registry. Pediatrics. 2008;122(5):e1100-e1107. 